\title{
Towards Optimization of Probe Placement for Radio-Frequency Ablation
}

Inga Altrogge ${ }^{1}$, Tim Kröger ${ }^{1}$, Tobias Preusser ${ }^{1}$, Christof Büskens ${ }^{2}$, Philippe L.Pereira ${ }^{3}$, Diethard Schmidt ${ }^{3}$, Andreas Weihusen ${ }^{4}$, and Heinz-Otto Peitgen ${ }^{1,4}$

${ }^{1} \mathrm{CeV}$ is - Center for Complex Systems and Visualization, University of Bremen, Germany

${ }^{2}$ ZETEM - Center for Industrial Mathematics, University of Bremen, Germany

${ }^{3}$ Dept. of Diagnostic Radiology, Eberhard Karls University, Tübingen, Germany

${ }^{4} \mathrm{MeVis}-$ Center for Medical Diagnostic Systems and Visualization, Bremen, Germany

\begin{abstract}
We present a model for the optimal placement of mono- and bipolar probes in radio-frequency ( $\mathrm{RF}$ ) ablation. The model is based on a numerical computation of the probe's electric potential and of the steady state of the heat distribution during RF ablation. The optimization is performed by minimizing a temperature based objective functional under these constraining equations. The paper discusses the discretization and implementation of the approach. Finally, applications of the optimization to artificial data and a comparison to a real $\mathrm{RF}$ ablation are presented.
\end{abstract}

\section{Introduction and Related Work}

During the last decade, local and minimally invasive techniques have become a promising treatment of tumor diseases. Especially in situations where a surgical resection or a chemotherapy is not possible due to the extent of the tumor or the general state of the patient, these techniques offer an alternative and less invasive possibility of treatment. Several authors [1, 2, 3, 4, 5, 6, have worked on the simulation of various thermo-therapy approaches (intervention methods where the malignant tissue is destroyed by high or low temperatures). The complexity of the models varies from simple steady state models with constant material parameters 2 to models taking vaporization of water and the nonlinear behavior of tissue properties into account $[\underline{6}$. The models are discretized by finite differences or finite element methods on Cartesian or tetrahedral grids.

In this work we consider the radio-frequency $(\mathrm{RF})$ ablation of liver tumors with mono- or bipolar systems: A probe, connected to an electric generator, is placed inside the malignant tissue, such that an electric current flows through the body and heats the tissue near the probe up to temperatures of more than $60{ }^{\circ} \mathrm{C}$. At such temperatures the proteins of the heated tissue coagulate and its cells die. The treatment is successful, if the volume of destroyed tissue completely includes the malignant lesion, comparable to R0 resections in liver surgery.

Because of local cooling due to blood perfusion a thorough planning of the probe's position as well as a determination of optimal generator power are advisable to assert a successful treatment. In this paper we take a first step towards 
an optimization of the RF ablation planning. For a steady state partial differential equation (PDE) model we formulate an appropriate objective functional measuring the quality of an ablation. Therewith, we aim at an optimization of the RF probe's positioning under the constraining PDE system. In a future work the optimal control of the electric energy induced into the malignant tissue will be investigated.

To our knowledge, the optimization of mono- and bipolar RF ablation in three space dimensions has not been incorporated in the existing finite element models. However, for interstitial ultrasound thermo-therapy, a two-dimensional optimization model has been presented by Bustany et al. 7]. Also, a parameter identification for the hyperthermia therapy is investigated by Gänzler et al. 8] . Moreover, Villard et al 9] presented a three-dimensional optimization based on a simple estimation of the lesion zone as an ellipsoid. Our problem falls in the field of nonlinear optimization subject to infinite dimensional constraints given by a system of PDEs. For an overview of the methodology see e. g. [10, 11.

The approach presented in the current paper is the result of a joint effort of $\mathrm{CeVis}$, MeVis and ZETEM at the University of Bremen and a variety of clinical partners working together in publicly funded research projects 1 . These projects focus on the development and clinical evaluation of computer aided systems for planning, monitoring, and assessing of tumor ablation. At CeVis, a group, headed by the third author, works on the advanced numerical models and algorithms.

\section{A Model for the Simulation of RF Ablation}

We consider the computational domain to be a cuboid $\Omega \subset \mathbb{R}^{3}$ with boundary $\Gamma_{\text {out }}=\partial \Omega$ in which a tumor $\Omega_{\mathrm{tu}} \subset \Omega$ lies. Further, we assume that a mono- or bipolar RF probe is applied in $\Omega$, whose position $\bar{x} \in \Omega$ (of the active zone's center) and direction $\bar{a} \in S^{2}=\left\{x \in \mathbb{R}^{3}:|x|=1\right\}$ are the unknown optimization variables. The subset of $\Omega$ that is covered by the probe is denoted by $\Omega_{\mathrm{pr}}$, the subsets covered by the electrodes are denoted by $\Omega_{+}$and/or $\Omega_{-}$, and $\Omega_{ \pm}=$ $\Omega_{+} \cup \Omega_{-}$(cf. Fig. 11). We emphasize that all these sets depend on $\bar{x}$ and $\bar{a}$.

In order to find the optimal values for $\bar{x}$ and $\bar{a}$, we must be able to solve the forward problem, i.e. to compute the temperature distribution resulting from the ablation for a given constellation $(\bar{x}, \bar{a})$. Hence, we are interested in the temperature $T$ and the electric potential $\varphi$ both of which are functions $\bar{\Omega} \rightarrow \mathbb{R}$. These functions are solutions of the following PDEs in $\Omega$ :

$$
\begin{aligned}
& -\nabla \cdot(\sigma \nabla \varphi)=0, \\
& -\nabla \cdot(\lambda \nabla T)=Q,
\end{aligned}
$$

Equation (1a) is the electrostatic potential equation derived from Maxwell's equations (cf. Stein [1]). Equation $(1 \mathrm{bb})$ is the steady state of the heat equation,

${ }^{1}$ LITT/RFITT funded by the German Research Foundation (Pe 199/11-1 and Pe 199/15-2) and the national research networks VICORA and FUSION funded by the Federal Ministry of Education and Research (01EZ0401 and 01IBE03C). 


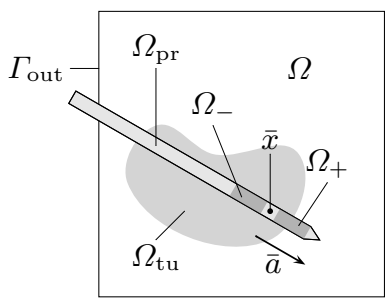

Fig. 1. Schematic setting of the considered configuration. Note that $\Omega_{+} \cup \Omega_{-}=\Omega_{ \pm} \subset \Omega_{\mathrm{pr}}$, where all these sets depend on $\bar{x}$ and $\bar{a}$.

where the right hand side $Q$ consists of the source $Q_{\mathrm{rf}}$ due to the electric current and the heat sink $Q_{\text {perf }}$ due to the blood perfusion. The electric and thermal conductivities $\sigma, \lambda \in \mathbb{R}$ are assumed to be constant. Thus, we have a system of PDEs, coupled by the right hand side of the heat equation.

Both equations are considered in $\Omega \backslash \overline{\Omega_{ \pm}}$and $\Omega$, respectively, together with the boundary conditions

$$
\begin{array}{rlrlrl}
\varphi & = \pm 1 & \text { on } \overline{\Omega_{+}} \text {and } \overline{\Omega_{-}} & \text {(fixed potential on electrodes), } \\
n \cdot \nabla \varphi & =\frac{n \cdot(\bar{x}-x)}{|\bar{x}-x|^{2}} \varphi & & \text { on } \Gamma_{\text {out }} & \text { (Robin boundary conditions), } \\
T & =T_{\text {body }} & \text { on } \Gamma_{\text {out }} & \text { (no effect apart from the probe), }
\end{array}
$$

where $n$ denotes the outer normal. Condition $(2 \mathrm{~b})$ is based on the idea that on the (outer) boundary (i. e. far away from the probe), the potential behaves approximately as induced by a point load at the barycenter $\bar{x}$ of the probe.

The source term $Q_{\mathrm{rf}}$ is, up to a scalar multiple, equal to the electric power $P=\sigma|\nabla \varphi|^{2}$, i. e.

$$
Q_{\mathrm{rf}}=\frac{P_{\text {eff }}}{P_{\text {total }}} P \quad \text { with } \quad P_{\text {total }}=\int_{\Omega} P d x, \quad P_{\text {eff }}=\frac{4 P_{\text {setup }} R R_{\mathrm{I}}}{\left(R+R_{\mathrm{I}}\right)^{2}}, \quad R=\frac{U^{2}}{P_{\text {total }}} .
$$

$R_{\mathrm{I}}$ is a property of the generator (inner resistance), $U$ is the difference of the potential $\varphi$ on the two electrodes $(U=2 \mathrm{~V}$ for bipolar probes and $U=1 \mathrm{~V}$ for monopolar probes), and $P_{\text {setup }}$ is the value set up at the generator's control unit.

Finally, the term modeling the cooling effects of the blood perfusion $Q_{\text {perf }}$ is based on the approach of Pennes [12]:

$$
Q_{\text {perf }}=-\nu \cdot\left(T-T_{\text {body }}\right) \quad \text { where } \nu= \begin{cases}\nu_{\mathrm{B}}^{*} \cdot \rho_{\mathrm{m}, \mathrm{B}} \cdot c_{\mathrm{m}, \mathrm{B}}, & \text { inside vessels } \\ 0, & \text { else } .\end{cases}
$$

$\nu$ depends on the relative blood circulation rate $\nu_{\mathrm{B}}^{*}\left[\mathrm{~s}^{-1}\right]$, the blood density $\rho_{\mathrm{m}, \mathrm{B}}[\mathrm{kg} / \mathrm{m}]$ and the heat capacity $c_{\mathrm{m}, \mathrm{B}}[\mathrm{J} / \mathrm{kg} \mathrm{K}]$. Actually, $Q_{\text {perf }}$ is split up into $\nu T_{\text {body }}$ (right hand side) and $\nu T$ (shifted to the left hand side), thus changing the differential operator in (1b).

\section{Objective Function}

The aim of the therapy is the destruction of the tumor with minimum amount of affected native tissue. In this work, we focus on a temperature based objective functional, i.e. for an optimal ablation the temperature shall be high in the 
region of the tumor $\Omega_{\mathrm{tu}}$ and near body temperature in the native tissue $\Omega \backslash \Omega_{\mathrm{tu}}$. Therefore the objective functional $f \in C^{1}\left(H^{1}(\Omega), \mathbb{R}\right)$ is chosen as

$$
f(T)=\frac{w_{1}}{2}\left\|T-T_{\text {body }}\right\|_{L^{2}\left(\Omega \backslash \Omega_{\mathrm{tu}}\right)}^{2}+\frac{w_{2}}{2}\left\|T-T_{\text {crit }}\right\|_{L^{2}\left(\Omega_{\mathrm{tu}}\right)}^{2}
$$

with $w_{1}$ and $w_{2}$ being suitable weights, so that the above requirements are met. Alternative approaches with objective functionals (cf. also 7]) not penalizing temperatures larger than $T_{\text {crit }}$ inside the tumor are currently investigated. Also, coagulation based objective functionals measuring the distance between the tumor $\Omega_{\mathrm{tu}}$ and the volume of coagulated tissue, are under consideration.

\section{Optimization Algorithm}

Formally, the objective function $f$ can be considered as a function of the temperature distribution $T$, where $T$ is a function of the heat source $Q_{\mathrm{rf}}$, and $Q_{\mathrm{rf}}$ is a function of the optimization parameter $(\bar{x}, \bar{a})=: u \in U=\Omega \times S^{2}$. Hence,

$$
Q_{\mathrm{rf}}=\mathcal{Q}(u), \quad \mathcal{Q} \in C^{1}\left(U, L^{2}(\Omega)\right), \quad T=\mathcal{T}\left(Q_{\mathrm{rf}}\right), \quad \mathcal{T} \in C^{1}\left(L^{2}(\Omega), H^{1}(\Omega)\right) .
$$

Thus, we are looking for $u \in U$ such that $F: U \rightarrow \mathbb{R}, u \mapsto F(u):=f \circ \mathcal{T} \circ \mathcal{Q}(u)$ becomes minimal. Obviously, in certain situations the uniqueness of a minimizing configuration is not guaranteed, e.g. for spherical tumors. This situation may also occur in practice for liver tumors which in general have a spherical-like shape. However, such a symmetry is broken by the consideration of surrounding blood vessels and their cooling effects. Moreover, for practical reasons the uniqueness of a solution is not needed and even local minima give important information about good probe and generator configurations. In a future model we will incorporate constraints for the optimization parameters which break any existing symmetry even further. Such constraints are given by anatomical structures (bones, colon, diaphragm) that must not be punctured during the ablation.

For the optimization, i. e. the minimization of $F$, we use a gradient descent method. More sophisticated approaches, in particular Lagrange-Newton (SQP) methods providing superlinear convergence, are considered in an ongoing work. The current choice is due to the fact that in our case the involved Hessian turns out to be very ill-conditioned.

Thus, we construct a series $\left(u^{n}\right)_{n \in \mathbb{N}} \subset U$ where $u^{0} \in U$ is chosen arbitrarily and $u^{n+1}=u^{n}+s^{n} v^{n}$. Here, $v^{n} \in \mathbb{R}^{6}$ is the descent direction which is chosen to be an approximation of $-\nabla_{u} F\left(u^{n}\right)$, and $s^{n}>0$ is the step size. We determine the step size by first trying $s^{n}=1$ and then bisecting it repeatedly until the resulting $u^{n+1}$ fulfills $u^{n+1} \in U$ and $F\left(u^{n+1}\right)<F\left(u^{n}\right)$. The iteration continues until $\left\|v^{n}\right\|_{L^{2}(\Omega)}$ falls below a given threshold.

The remaining part of this section deals with our approximation of the six components $\partial_{u_{j}} F(u)$ of the gradient $\nabla_{u} F(u)$. Using the chain rule yields

$$
\begin{aligned}
\partial_{u_{j}} F(u) & =f^{\prime}(\mathcal{T} \circ \mathcal{Q}(u))\left[\mathcal{T}^{\prime}(\mathcal{Q}(u))\left[\partial_{u_{j}} \mathcal{Q}(u)\right]\right] \\
& =\mathcal{T}^{\prime}(\mathcal{Q}(u))^{*}\left[f^{\prime}(\mathcal{T} \circ \mathcal{Q}(u))\right]\left[\partial_{u_{j}} \mathcal{Q}(u)\right]
\end{aligned}
$$


where ${ }^{\prime}$ denotes the Fréchet derivative, * denotes the adjoint operator, and brackets [.] denote application of linear operators. For the calculation of $\partial_{u_{j}} \mathcal{Q}(u)$ we use a numerical approximation by central differences, whereas we determine $f^{\prime}$ analytically as

$$
\begin{aligned}
& f^{\prime}(T)[\tilde{T}]=w_{1}\left\langle T-T_{\text {body }}, \tilde{T}\right\rangle_{L^{2}\left(\Omega \backslash \Omega_{\mathrm{tu}}\right)}+w_{2}\left\langle T-T_{\text {crit }}, \tilde{T}\right\rangle_{L^{2}\left(\Omega_{\mathrm{tu}}\right)}=\left\langle\alpha_{T}, \tilde{T}\right\rangle_{L^{2}(\Omega)} \\
& \text { with } \alpha_{T} \in L^{2}(\Omega) \text { given by } \quad \alpha_{T}(x)= \begin{cases}w_{1}\left(T(x)-T_{\text {body }}\right) & \text { for } x \in \Omega \backslash \Omega_{\mathrm{tu}} \\
w_{2}\left(T(x)-T_{\text {crit }}\right) & \text { else }\end{cases}
\end{aligned}
$$

For $\mathcal{T}^{\prime}(\cdot)^{*}$ a straightforward computation leads to the representation

$$
\mathcal{T}^{\prime}\left(Q_{\mathrm{rf}}\right)^{*}\left[f^{\prime}(T)\right]\left[\tilde{Q}_{\mathrm{rf}}\right]=\left\langle p_{T}, \tilde{Q}_{\mathrm{rf}}\right\rangle_{\Omega}
$$

where $p_{T} \in H^{1}(\Omega)$ is the solution to the adjoint problem (1b), (2c):

$$
-\lambda \Delta p_{T}+\nu p_{T}=\alpha_{T} \quad \text { in } \Omega, \quad p_{T}=0 \quad \text { on } \Gamma_{\text {out }},
$$

remember the term $\nu p_{T}$ is due to the splitting of $Q_{\text {perf }}$ introduced in Sect. 2 .

\section{Discretization with Finite Elements}

The solutions of the elliptic boundary value problems (1) and (4) are numerically computed with a finite element method on a three dimensional uniform Cartesian grid. The electric power $P=\sigma|\nabla \varphi|^{2}$, which is needed to calculate the right hand side $Q$ for (1b), is determined with central differences after the solution of (1a).

For reasons of analogy we restrict the following description to the elliptic problem (1b) which we assume to be adjusted to homogeneous boundary conditions in the usual way (see Braess [13]). We obtain the weak form, by multiplying the corresponding PDE with a test function $v \in H_{0}^{1}(\Omega)$. Integrating by parts over $\Omega$ leads to

$$
\lambda\langle\nabla T, \nabla v\rangle_{L^{2}(\Omega)}+\nu\langle T, v\rangle_{L^{2}(\Omega)}=\left\langle Q_{\mathrm{rf}}+\nu T_{\mathrm{body}}, v\right\rangle_{L^{2}(\Omega)} \quad \forall v \in H_{0}^{1}(\Omega) .
$$

In a second step we discretize this variational problem by covering the computational domain $\Omega$ with a uniform Cartesian grid. For that purpose we restrict (5) on a finite dimensional subspace $V^{h} \subset H_{0}^{1}(\Omega)$ consisting of piecewise trilinear, globally continuous functions. Then every function $w \in V^{h}$ is determined by its nodal values $\gamma_{i}$ at the vertices $x_{i}$ for $i=1, \ldots, n$ of the grid. This leads to a system of linear equations, which we solve with a conjugate gradient method, implemented in a matrix free way.

\section{$6 \quad$ Numerical Results}

In this section we present the application of our optimization to artificial settings as well as to geometries obtained from real CT scans. Let us first verify the performance of the algorithm in a case where the correct solution is qualitatively 

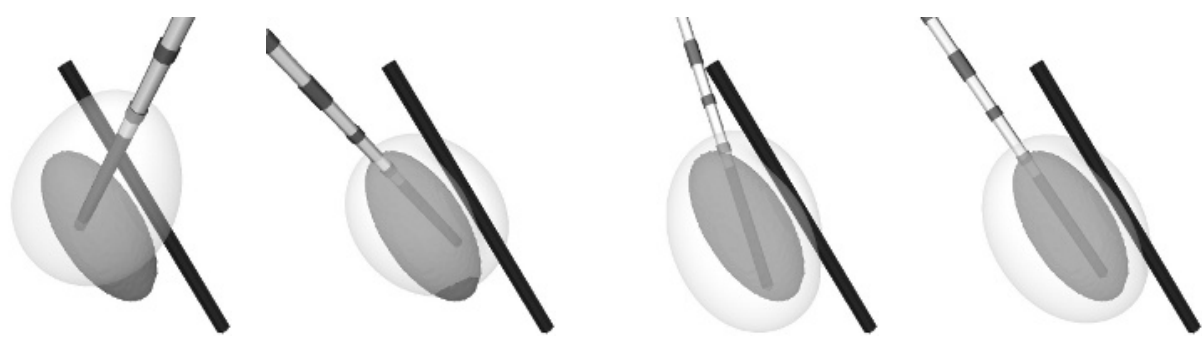

Fig. 2. Optimization of the probe's placement for an artificial example with an ellipsoid-shaped tumor (dark gray) and a linear vessel (black). The pictures show from left to right steps 0 (start), 4, 6, and 9 of the optimization and display the probe (white-gray) and a corresponding isosurface of the temperature (transparent) as well.

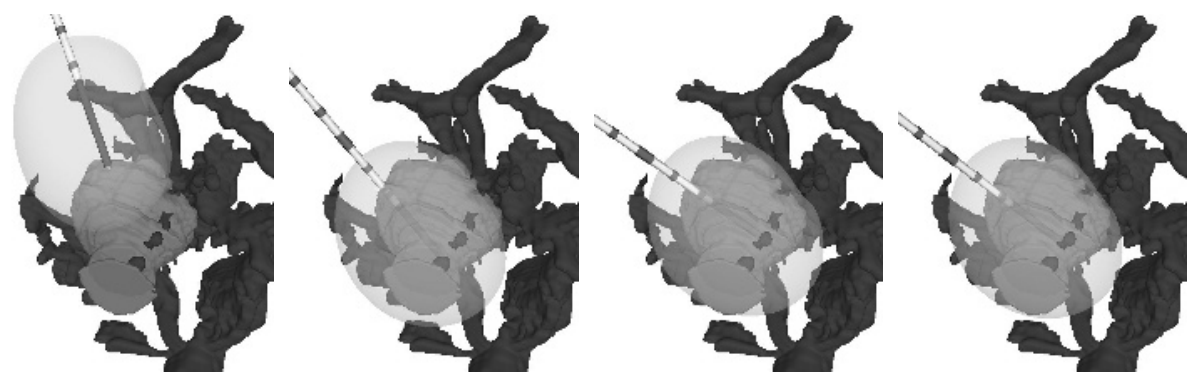

Fig. 3. Optimization for an example based on patient data with segmented tumor and surrounding vessels. The pictures show from left to right steps $0,5,10$ and 15 of the optimization process and display the tumor together with the vascular system, the probe position and an isosurface of the temperature (cf. Fig. 2).

obvious. We let $\Omega$ be a domain of extent $60 \times 60 \times 60\left[\mathrm{~mm}^{3}\right]$ with an overlaid grid consisting of 121 grid points in each direction. As a tumor we consider an ellipsoid, which lies in the center of $\Omega$, together with a straight vessel in the vicinity of the tumor (cf. Fig. 2). A monopolar probe with a radius of $1.2 \mathrm{~mm}$ and an electrode length of $25 \mathrm{~mm}$ is used. The generator has an inner resistance of $80 \Omega$ and is set up to a power of $80 \mathrm{~W}$. The initial probe position for the optimization is located at $10 \mathrm{~mm}$ distance in each direction from the center of $\Omega$ and its orientation is $\bar{a}=(5,2,3)$ normalized to length 1 . The results of this experiment are shown in Fig. 2. It is clearly visible that the probe placement adapts to the ellipsoidal shape of the tumor and thus corresponds to the expected positioning along the long principal axis of the ellipsoid.

The second example is performed with a real segmented tumor and a set of surrounding vessels. The computational domain $\Omega$ has a dimension of $121.4 \times$ $80.7 \times 80.7\left[\mathrm{~mm}^{3}\right]$ and the overlaid grid consists of $155 \times 103 \times 103$ grid points. The probe data is the same as in the first example, whereas the generator settings are $R_{\mathrm{I}}=80 \Omega$ and $P_{\text {setup }}=30 \mathrm{~W}$. Moreover, the initial position of the probe is $(30.7,10.3,10.3) \mathrm{mm}^{3}$ distant from the center of $\Omega$ and the probe's initial 


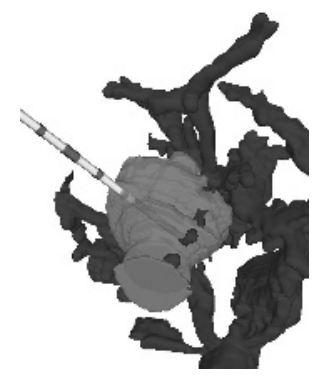

Fig. 4. Comparison of the computed optimal probe placement (left) with the probe placement chosen by the responsible physician (right)

direction is $\bar{a}=(5,2,3)$ normalized to 1 . As the pictures in Fig. 3 show, the optimal probe's position lies in the center of the tumor, and its optimal direction adapts to the shape of the tumor as well as to the influence of the vessels. The cooling effect of the blood perfusion can be seen explicitly on the steady state of the temperature (i. e. a lower temperature near the vessels).

Finally we compare the result of our second optimization to the positioning chosen by the responsible physician. Instead of one probe of radius $1.2 \mathrm{~mm}$, the physician had taken a fixed cluster of three parallel probes of radius $0.75 \mathrm{~mm}$. For the time-being we assume that these act like a single probe of larger radius. Although this is only a rough approximation, the result depicted in Fig. 4 shows that the probe placement suggested by our algorithm and the one chosen by the physician nearly coincide in their position (i.e. in the barycenter of the probes' active zones), whereas the orientation differs by an angle of about $37^{\circ}$. One reason for this could be the spherical-like shape of the tumor - an intrinsic property of the problem (cf. Sect. 4). Also, anatomical structures (e. g. bones or internal organs), which are not respected in our model yet, could have excluded the orientation found by our algorithm from the physician's choice.

\section{Discussion and Future Work}

We have discussed a model for the optimization of the placement of mono- and bipolar probes in RF ablation. The algorithm has been evaluated on an artificial example for which the optimal probe's positioning is known. Furthermore, results of the optimization with a CT-segmented tumor surrounded by a vascular system have been presented and compared to the probe's placement the responsible physician had chosen for his ablation. Although the computed optimal probe's direction differs due to e.g. anatomical structures (cf. Sect. 6), the probe's position is similar to the physician's choice.

We regard the results presented in this work as useful for the planning of RF ablations as well as for educational purposes, since they help understanding how experienced physicians choose their RF ablation parameters.

To provide practically feasible optimal positioning (respecting structures that must not be punctured), we are planning to incorporate state constraints for the optimization parameters in a future model. We regard the optimization of probe placement as a helpful tool for tumor ablation, in particular with respect to 
the complete destruction of the tumor lesion. A correct placement of the probe increases the chance of a total tumor destruction and therefore reduces the risk of a recurrence. In ongoing and future investigations, we intend to incorporate sophisticated Lagrange-Newton and active set strategies for the optimization. Moreover, we will consider the corresponding time-dependent PDE models and work towards an optimal control of the overall energy deposit during ablation. In practice it will be hard for the interventionist to achieve the computed optimal positions exactly. Inevitably there will be deviations from the optimal configuration, for which a parametric sensitivity analysis is going to provide an estimation of the risk of failure of the therapy. Finally more comprehensive evaluation with artificial and real data sets (different tumor shapes and vessel configurations) will also be performed.

The authors thank the VICORA team and in particular T. Stein and A. Roggan from Celon AG for valuable hints and fruitful discussions on the topic. Also, we would like to thank the team from MeVis, especially S. Zentis and C. Hilck for preprocessing the CT scans.

\section{References}

1. Stein, T.: Untersuchungen zur Dosimetrie der hochfrequenzstrominduzierten interstitiellen Thermotherapie in bipolarer Technik. Volume 22 of Fortschritte in der Lasermedizin. Müller and Berlien (2000)

2. Tungjitkusolum, S., Staelin, S.T., Haemmerich, D., et al.: Three-dimensional finiteelement analyses for radio-frequency hepatic tumor ablation. IEEE Trans. Biomed. Eng. 49 (2002) 3-9

3. Welp, C., Werner, J.: Large vessel cooling in hepatic radiofrequency ablation: investigation on the influence of blood flow rate. IEEE Trans. Biomed. Eng. (submitted)

4. Deuflhard, P., Weiser, M., Seebaß, M.: A new nonlinear elliptic multilevel FEM applied to regional hyperthermia. Comput. Vis. Sci. 3 (2000) 115-120

5. Roggan, A.: Dosimetrie thermischer Laseranwendungen in der Medizin. Volume 16 of Fortschritte in der Lasermedizin. Müller and Berlien (1997)

6. Kröger, T., Altrogge, I., Preusser, T., Peitgen, H.O., et al.: Numerical simulation of radio frequency ablation with state dependent material parameters in three space dimensions. In: Proceedings of MICCAI. (2006) submitted.

7. Khalil-Bustany, I.S., Diederich, C.J., Polak, E., Kirjner-Neto, C.: Minmax optimization-based inverse treatment planning for interstitial thermal therapy. Int. J. Hyperthermia 14 (1998) 347-366

8. Gaenzler, T., Volkwein, S., Weiser, M.: SQP methods for parametric identification problems arising in hyperthermia. Optimization Methods and Software (2004)

9. Villard, C., Soler, L., Gangi, A.: Radiofrequency ablation of hepatic tumors: simulation, planning, and contribution of virtual reality and haptics. Computer Methods in Biomechanics and Biomedical Engineering 8(4) (2005) 215-227

10. Tröltzsch, F.: Optimale Steuerung partieller Differentialgleichungen. Vieweg (2005)

11. Geiger, C., Kanzow, C.: Theorie und Numerik restringierter Optimierungsaufgaben. Springer, Berlin (2002)

12. Pennes, H.H.: Analysis of tissue and arterial blood temperatures in a resting forearm. J. Appl. Physiol. 1 (1948) 93-122

13. Braess, D.: Finite Elemente. 3rd edn. Berlin, Springer (2003) 